\title{
Growth Plate, Bone and Mineral Metabolism
}

\author{
Outi Mäkitiea, Agnès Linglart ${ }^{b}$ and Terhi J. Heinoc \\ aPediatric Endocrinology and Metabolic Bone Diseases, Children's Hospital, Helsinki University Central Hospital \\ and University of Helsinki, Helsinki, Finland \\ ${ }^{b}$ Department of Endocrinology and Diabetes for Children, Center of Reference for Rare Disorders of Calcium \\ and Phosphorus Metabolism, Hôpital Bicêtre, Le Kremlin-Bicêtre, France \\ cDepartment of Cell Biology and Anatomy, University of Turku, Turku, Finland
}

\begin{abstract}
Recent substantial advances in molecular genetic methodology have led to a rapid expansion of our knowledge and understanding of molecular mechanisms governing normal skeletal growth and homeostasis. The complexity of the regulatory networks and the number of players therein is remarkable. Several key pieces in this puzzle have been discovered by studies involving children with rare skeletal diseases, such as osteogenesis imperfecta or other skeletal dysplasias, or children with disturbed mineral metabolism, such as hypophosphatemic rickets. Understanding the pathophysiology and the molecular mechanisms leading to skeletal or mineral disorders provides a solid background for the development of specific therapies.

During the past year several new genes in various skeletal conditions have been discovered, as seen in the selected papers. However, equally exciting is to see the increasing number of studies describing attempts to find therapies for these skeletal disorders, such as achondroplasia and hypochondroplasia caused by overactive FGF receptor, or hypophosphatemic disorders caused by increased FGF23. As shown in this chapter, the preliminary results are promising, but much work remains to be done before these therapies are available for patient care. While research on pathological conditions moves forward, several aspects in the skeletal physiology still remain poorly understood: How do bones grow? How do growth plate cells function without oxygen (or with little oxygen)? And what is the role of the osteocytes, the quiet bone cells entrapped within the mineralized matrix?
\end{abstract}

\section{Mechanism of the year \\ How chondrocytes grow bigger?}

\section{Multiple phases of chondrocyte enlargement underlie differences in skeletal proportions}

Cooper KL, Oh S, Sung Y, Dasari RR, Kirschner MW, Tabin CJ

Department of Genetics, Harvard Medical School, Boston, MA, USA

kcooper@genetics.med.harvard.edu

Nature 2013:495:375-378

Background: There is a wide diversity of skeletal proportions in different mammals and furthermore, each individual is comprised of a variety of bones of differing lengths. It is known that the enlargement of hypertrophic chondrocytes in the growth plate mostly contributes to elongation of an individual bone but the mechanisms for cell enlargement are still unknown.

Methods: The authors used quantitative phase microscopy to analyze hypertrophic chondrocytes in tibia, radius, metacarpi and metatarsi of fetal mice and jerboa pups.

Results: Hypertrophic chondrocytes were shown to increase in volume through three distinctive phases. When analyzing mouse bones, rapidly elongating tibia had large hypertrophic chondrocytes, while in the slowly elongating proximal radius smaller cells were found. Jerboa (a bipedal rodent with greatly elongated hind limbs) had only slightly larger hypertrophic chondrocytes in proximal tibia when compared to mice. The greatest differences between the two species were observed in metatarsals: hypertrophic chondrocytes in jerboa were $58 \%$ higher and the cells increased their volume almost 40 -fold from their initial volume when compared to mouse cells. In addition, IGF-1-deficient mice had smaller hypertrophic chondrocytes, even though the cell numbers were the same as in wild-type animals. 
Conclusion: Mammalian chondrocytes undergo three distinct phases of volume increase, including a phase of massive cell swelling in which the cellular dry mass was significantly diluted. Cell swelling is the main mechanism for hypertrophic chondrocyte enlargement. The most varying parameter between the rapidly and slowly elongating growth plates was the duration of the final phase of this volume enlargement. Moreover, this third phase was locally regulated through an IGF-1-dependent mechanism. This study provides a framework for understanding the regulation of skeletal size and for exploring how cells could sense, modify and establish a volume set-point.

Each long bone in the embryo forms via the endochondral ossification process, in which multiple cellular events, including chondrocyte proliferation, matrix production and finally hypertrophic chondrocyte enlargement take place. It is remarkable that each mammalian species has distinguished skeletal proportions and that in addition each individual has a great variety of bones of differing sizes and shapes. Genetic determinants and mechanical loading most certainly play a role here but what is the cellular mechanism giving rise to these dramatic differences? According to current understanding, largest contribution to the lengthening of a skeletal element appears to come from a dramatic increase in the volume of hypertrophic chondrocytes as they undergo terminal differentiation [1].

The current study by Cooper et al. aimed at looking one step deeper: dissecting the detailed mechanisms for hypertrophic chondrocyte volume enlargement. By using a relatively simple approach but a sophisticated method of diffraction phase microscopy, they could demonstrate that hypertrophic chondrocytes can enlarge extremely rapidly by the coordinated multiphase process of cell swelling. This was shown to lower the energetic cost of growth and was further amplified by the anabolic actions of IGF-1. Analysis of mouse vs. jerboa bones further demonstrated the evolutionary basis for their findings: differences in chondrocyte enlargement during fetal bone development were directly reflected in the final size of bones. This study provides a new mechanistic view for understanding how our skeletal size is regulated. It furthermore gives a deeper insight into the way cells sense, modify and establish volumetric set-points to fulfill a certain function during their life-span.

\section{New mechanism \\ A novel role for HIF-1 $\alpha$ in collagen production during hypoxia}

\section{HIF1 $\alpha$ is a central regulator of collagen hydroxylation and secretion under hypoxia during bone development}

Bentovim L, Amarilio R, Zelzer E

Department of Molecular Genetics, Weizmann Institute of Science, Rehovot, Israel

eli.zelzer@weizmann.ac.il

Development 2012;139:4473-4483

Background: Collagen production is an essential process in all multicellular organisms. It depends on hydroxylation of proline residues, a reaction that uses molecular oxygen as a substrate. Paradoxically, growth plate chondrocytes produce vast amounts of collagen in avascular, hypoxic conditions.

Methods: Cartilage-specific knockout mice were generated by crossing floxed-Hif1 $\alpha$ mice with Col2-CreET ${ }^{\mathrm{T}}$ mice, allowing a temporary knockout by tamoxifen treatment. Developing embryonic bones were analyzed by various methods and the results were confirmed with in vitro studies on isolated chondrocytes. Results: Transcription factor HIF1 $\alpha$ was identified as a central component in a mechanism that underlies collagen hydroxylation and secretion by hypoxic cells. Hif $1 \alpha$ loss-of-function in growth plate chondrocytes arrested the secretion of extracellular matrix proteins, including collagen type II. Reduced collagen hydroxylation and endoplasmic reticulum stress induction in Hif1 $\alpha$-depleted cells suggested that HIF1 $\alpha$ regulates collagen secretion by mediating its hydroxylation and consequently folding. Hif $1 \alpha$ was shown to induce transcription of collagen prolyl-4-hydroxylase, which catalyzes collagen hydroxylation. Furthermore, the results demonstrated that HIF1 $\alpha$ maintained cellular levels of oxygen, most likely by controlling the expression of pyruvate dehydrogenase kinase 1 .

Conclusion: Through these mechanisms, HIF1 $\alpha$ acts as a central regulator of collagen production that allows chondrocytes to maintain their function as professional secretory cells in the hypoxic growth 
plate. Hypoxic conditions are also known to occur during pathological conditions such as cancer, indicating that besides embryogenesis, these findings may also promote our understanding of various pathological processes.

Growth plates, which are located at the two ends of developing bones, usually contain low levels of oxygen [2]. Chondrocytes located within this specialized tissue are secretory cells producing large amounts of extracellular matrix (ECM), including collagen type II, as well as non-collagenous components, such as proteoglycans. One of the crucial steps in the biosynthesis of ECM proteins is their folding in the endoplasmic reticulum (ER), which usually depends on their posttranslational modifications. For example, a key step in collagen production is the hydroxylation of proline residues, a reaction, which is catalyzed by a group of enzymes known as collagen prolyl-4-hydroxylases [3]. This paradigm of hypoxic conditions at the growth plate and on the other hand the chondrocytes' need for oxygen-dependent proline hydroxylation was the basis of the current study.

The authors took advantage of a mouse model, in which a known hypoxia-induced transcription factor, HIF1 $\alpha$, was knocked out in a cartilage-specific temporarily controlled manner. They were able to demonstrate that HIF1 $\alpha$ is a central component in a mechanism that allows collagen hydroxylation to occur even in hypoxic cells. HIF1 $\alpha$ was shown to both increase the catalyst (collagen prolyl-4-hydroxylases) and to maintain the substrate (oxygen) levels. In the absence of HIF1 $\alpha$, enzyme subunit expression and intracellular oxygen levels were reduced, leading to reduced collagen type II hydroxylation and its impaired secretion and intracellular accumulation. This mechanism appears to furthermore be related to the phenomenon called ER stress, which has recently been shown to be important in the chondrocyte differentiation during bone development [4]. In summary, besides the 'traditional' role of HIF1 $\alpha$ in upregulating various genes to promote survival in low-oxygen conditions, this factor can also directly maintain the posttranslational modifications of collagen in chondrocytes and thereby regulate the ECM secretion.

Food for thought

Tick-tack-tick-tack: clock genes regulate bone growth

\title{
Clock genes influence gene expression in growth plate and endochondral ossification in mice
}

\author{
Takarada T, Kodama A, Hotta S, Mieda M, Shimba S, Hinoi E, Yoneda Y \\ Laboratory of Molecular Pharmacology, Division of Pharmaceutical Sciences, Kanazawa University Graduate School, \\ Kanazawa, Japan
}

yyoneda@p.kanazawa-u.ac.jp

J Biol Chem 2012;287:36081-36095

Background: The authors have previously shown transient promotion of Period-1 (Per1) gene expression by parathyroid hormone (PTH) in cultured chondrocytes. The aim of the current study was to investigate the modulation of chondrogenic differentiation by clock genes and to elucidate the role of Indian hedgehog (Ihh) in the circadian regulation of growth plate chondrocyte differentiation and longitudinal bone growth in vivo.

Methods: Various animal models and cell culture assays were applied.

Results: Several clock genes were demonstrated to be expressed with oscillatory rhythmicity in cultured chondrocytes and rib growth plate of mice. On the other hand, chondrogenesis was markedly inhibited in stable transfectants of Per1 both in chondrocytic ATDC5 cells and in rib growth plate chondrocytes from mice deficient of brain and muscle aryl hydrocarbon receptor nuclear translocator-like (BMAL1). Different clock gene products were shown to regulate Ihh promoter activity and a clear circadian rhythmicity in Ihh expression profiles was observed in the growth plate. BMAL1-null mice had a smaller body size and shorter bones than wild-type mice, which was associated with a significant decrease of Ihh expression in the growth plate. Growth plate chondrocytes from null mice had impaired chondrogenic potential and disrupted rhythmic expression profiles of Per1 and Ihh. Young mice with a chondrocyte-specific knockout of BMAL1 had similar abnormalities in bone growth and Ihh expression. 
Conclusion: Endochondral ossification appears to be under the control of certain clock gene products, which are expressed in chondrocytes during postnatal skeletogenesis. The mechanism involves direct regulation of the Ihh transactivation.

Circadian rhythm is known to regulate many physiological and pathological processes in various organisms from bacteria to mammals. Traditionally, circadian rhythm is thought to be centrally regulated by the cells of suprachiasmatic nucleus of the anterior hypothalamus [5], but it has been suggested that peripheral tissues could also be involved. Indeed, local expression of clock genes, such as Period (Per), has been demonstrated in various peripheral tissues [6] where the direct regulation of the oscillatory rhythm can take place.

Authors of the current paper had previously shown a transient upregulation of Per 1 in response to PTH in vitro [7] and now aimed to study the potential involvement of clock genes in the regulation of longitudinal bone growth in vivo. By using various knockout animal models and elegant cell culture experiments, which were complemented with detailed gene and protein expression studies, they were able to demonstrate a clear circadian rhythmicity in the expression profile of Ihh, the master regulator of chondrogenesis. This associated with the rhythmic expression of clock genes Per 1 and Bmal1. They concluded that rhythmic expression of Ihh leads to oscillated bone elongation during skeletogenesis, indicating a new mechanism for bone growth regulation. However, one should still remember that, besides cell autonomous effects, bone growth is also strongly regulated by circulating endocrine factors. One example is growth hormone, which is known to be rhythmically secreted by the anterior pituitary according to the sleep cycle. Therefore, it is likely that the effects of circadian rhythm on longitudinal bone growth can be mediated by a team of central, local as well as systemic players.

\section{New hope \\ Novel therapeutic agents for achondroplasia}

\section{Evaluation of the therapeutic potential of a CNP analog in a Fgfr3 mouse model recapitulating achondroplasia}

Lorget F, Kaci N, Peng J, Benoist-Lasselin C, Mugniery E, Oppeneer T, Wendt DJ, Bell SM, Bullens S, Bunting S, Tsuruda LS, O’Neill CA, Di Rocco F, Munnich A, Legeai-Mallet L

Marin Pharmaceutical, Novato, CA, USA, and Institut National de la Santé et de la Recherche Médicale Unité 781, Université Paris Descartes-Sorbonne Paris Cité, Institut Imagine, Hopital Necker-Enfants Malades, Paris, France laurence.legeai-mallet@inserm.fr

Am J Hum Genet 2012;91:1108-1114

Background: Achondroplasia (ACH) is the most common form of dwarfism. It is an inherited autosomaldominant chondrodysplasia caused by a gain-of-function mutation in fibroblast-growth-factor-receptor 3 (FGFR3). C-type natriuretic peptide (CNP) antagonizes FGFR3 downstream signaling and normalizes the dwarfism of $F g f r 3^{\text {ach/+ }}$ mice but therapeutic potential is limited by short half-life.

Methods: The authors designed and manufactured BMN 111, a 39-aa CNP analog with an extended plasma half-life due to its resistance to neutral-endopeptidase (NEP) digestion. The pharmacological activity of BMN 111 was evaluated by in vitro, ex vivo and in vivo systems, including human growth plate chondrocytes with an FGFR3 gain-of-function mutation, and Fgfr $3^{\mathrm{Y} 367 \mathrm{C} /+}$ mouse corresponding the human thanatophoric dysplasia mutation.

Results: In ACH human growth-plate chondrocytes, a decrease in the phosphorylation of ERKs 1 and 2 was demonstrated, thus confirming that BMN 111 inhibited FGF-mediated MAPK activation. The phenotype of $\mathrm{Fgfr} 3^{\mathrm{Y} 367 \mathrm{C} /+}$ mice showed $\mathrm{ACH}$-related clinical features and treatment with BMN 111 led to a significant recovery of bone growth. The authors observed an increase in the axial and appendicular skeleton lengths, and improvements in dwarfism-related clinical features included flattening of the skull, reduced crossbite, straightening of the tibias and femurs, and correction of the growth-plate defect.

Conclusion: These results provide the proof of concept that BMN 111, a NEP-resistant CNP analog, might benefit individuals with $\mathrm{ACH}$ and hypochondroplasia. 


\section{A novel FGFR3-binding peptide inhibits FGFR3 signaling and reverses the lethal phenotype of mice mimicking human thanatophoric dysplasia}

Jin M, Yu Y, Qi H, Xie Y, Su N, Wang X, Tan Q, Luo F, Zhu Y, Wang Q, Du X, Xian CJ, Liu P, Huang H, Shen Y, Deng CX, Chen D, Chen L

Center of Bone Metabolism and Repair, State Key Laboratory of Trauma, Burns and Combined Injury, Institute of Surgery Research, Dapital Hospital, Third Military University, Chongqing, China

linchen70@163.com

Hum Mol Genet 2012;21:5443-5455

Background: Achondroplasia, hypochondroplasia and thanatophoric dysplasia (TD) are human skeletal dysplasia syndromes, which are caused by gain-of-function mutations in fibroblast growth factor receptor-3 (FGFR3). TD is the most common form of skeletal dysplasia that is lethal in the neonatal period and has been classified into TDI and TDII. At present, there are no effective treatments available for these diseases.

Methods: In this study, a random 12-peptide phage library was screened using FGFR3 as bait, and 23 positive clones were obtained. They all shared an identical amino acid sequence (VSPPLTLGQLLS), named as peptide P3. This peptide had high binding specificity to the extracellular domain of FGFR3. The effects of this peptide were tested with cultured chondrocytes and TDII mouse metatarsals in vitro, as well as with TDII mouse in vivo.

Results: P3 inhibited tyrosine kinase activity of FGFR3 and its typical downstream molecules, ERK/ MAPK. It also promoted the proliferation and chondrogenic differentiation of cultured chondrogenic ATDC 5 cells and improved the metatarsal bone growth retardation in mice mimicking human TDII. In addition, P3 reversed the neonatal lethality of TDII mice and rescued the severe defects of growth plate development.

Conclusion: A novel inhibitory peptide for FGFR3 signaling was identified. It may serve as a potential therapeutic agent for the treatment of FGFR3-related skeletal dysplasias.

Activating FGFR3 mutations in humans are associated with several skeletal dysplasias, including achondroplasia (ACH) and thanatophoric dysplasia (TD). Currently, there are only few approaches that can be used to treat these patients; the most common being GH treatment and surgical limb lengthening. These methodologies are however limited in their outcomes: GH treatment has no clear long-term benefit and surgery is controversial and highly invasive. Furthermore, due to its neonatal lethality, surgical and GH treatment of TD patients is practically impossible. FGFR3 and its downstream pathways have therefore been identified as attractive targets for therapy. One candidate has been C-type natriuretic peptide (CNP), which is a potent stimulator of endochondral bone growth and was already several years ago identified as a potential means for rescuing $\mathrm{ACH}$ [8]. Because CNP is an intrinsic peptide, the safety of CNP therapy is potentially not a major concern. The major limitation of CNP, however, is its short half-life ( 2 min after i.v. administration) making its use very challenging in pediatric patients. Therefore, novel approaches with synthetic CNP analogs have been taken to achieve a longer half-life. One example of such an approach was discussed in Yearbook 2009, where a synthetic CNP-22 was shown to rescue ACH in mice without significant adverse effects [9].

Lorget et al. report a synthetic 39-aa CNP analog, which has extended plasma half-life due to its resistance to neutral endopeptidase, thus allowing once-a-day s.c. administration. They showed that 10 days of treatment with this peptide was able to partially rescue the $\mathrm{ACH}$ phenotype of $\mathrm{Fg} \mathrm{fr}^{\mathrm{Y}} 367 \mathrm{Cl}+$ mice by improving the dwarfism, confirmed by histological changes at growth plate expansion. The observed increase in prehypertrophic and hypertrophic zones and larger size of hypertrophic chondrocytes was consistent with the previous study using a 3-week continuous CNP-22 i.v. infusion [9]. Another type of approach was taken by Jin et al. who identified a specific FGFR3-binding peptide, P3, using a phage display technique. P3 was demonstrated to suppress the tyrosine kinase activity of FGFR3 and the FGFR3-mediated ERK/MAPK activation, and to promote chondrocyte proliferation and survival. They used a different mouse model mimicking human TDII and showed that P3 attenuated the FGFR3-mediated growth inhibition in mouse metatarsal bones in vitro. Intriguingly, the lethal phenotype of TDII mice could be rescued by injecting P3 to pregnant mice, which was accompanied by a relatively normal thoracic cavity and lung structure. This supports the concept that the respiratory distress in TD patients is caused by cartilage defects at their thoracic cavity. No gross histopathological changes were observed in other important organs of the mice treated with P3. Still, the results were not optimal since the body size remained smaller and the skull was still dome-shaped in the 
P3-treated TDII animals when compared to WT. In summary, more research is needed to confirm the long-term effectiveness and safety of the strategies presented in these two papers. In addition, direct targeting of therapeutic agents to growth plate cartilage may further enhance efficacy and minimize side effects of future therapies.

\section{New hope \\ A super-targeted therapy for osteopetrosis}

\section{Osteopetrosis rescue upon RANKL administration to Rankl ${ }^{--}$mice: a new therapy for human RANKL-dependent ARO}

Lo lacono N, Blair HC, Poliani PL, Marrella V, Ficara F, Cassani B, Facchetti F, Fontana E, Guerrini MM, Traggiai E, Schena F, Paulis M, Mantero S, Inforzato A, Valaperta S, Pangrazio A, Crisafulli L, Maina V, Kostenuik P, Vezzoni P, Villa A, Sobacchi $C$ UOS/IRGB, Milan Unit, CNR, Milan, and Humanitas Clinical and Research Center, Rozzano, Italy J Bone Miner Res 2012;27:2501-2510

Background: Autosomal recessive osteopetrosis (ARO) is usually treated with hematopoietic stem cell transplantation (HSCT). However, this approach is not effective in the rare osteoclast-poor form of ARO that is caused by mutations in the receptor activator of NF-кB ligand (RANKL) gene.

Methods: The authors studied the effects of replacement therapy with soluble recombinant murine RANKL (sRANKL) in a mouse model of RANKL-dependent ARO.

Results: The sRANKL was administered to $\mathrm{Rankl}^{-/-}$mice every $48 \mathrm{~h}$ starting early postnatally. Treatment with sRANKL for 1 month significantly improved the bone phenotype. While the $\mathrm{Rankl}^{-/}$mice had very dense bones, extremely limited marrow space, abnormally high mineralization, and complete absence of $\mathrm{TRAP}^{+}$cells, the $\mathrm{Rankl}^{-/-}$mice treated with sRANKL showed nearly complete rescue of the bone phenotype, with reduction in bone content, increase in marrow space, formation of vascular spaces, and only minimal retention of primary spongiosa. Re-establishment of osteoclast differentiation and function was demonstrated by marked TRAP staining in the bone. The increase in space within bone led to improvement of medullary hematopoiesis and beneficial effects on spleen and thymus. However, with higher doses and longer treatment, major adverse effects arose. The osteopetrotic phenotype was reversed to an osteopenic condition and detrimental changes in lung parenchyma led to early death in mice treated with high-dose sRANKL.

Conclusion: This study provides evidence that pharmacological administration of RANKL may provide an appropriate treatment option for RANKL-deficient ARO patients. The safety and optimal dosing need to be validated in a pilot clinical trial.

Autosomal recessive osteopetrosis (ARO) is a rare genetic bone disease presenting early in life with extreme sclerosis of the skeleton, reduction of bone marrow space, hepatosplenomegaly, anemia, compression of cranial nerves, and severe growth impairment. The disease is often lethal in early childhood. Hematopoietic stem cell transplantation (HSCT) remains the only treatment option. At least six genes have been implicated in the pathogenesis. Early identification of the underlying genetic defect is important for assessment of prognosis and treatment by HSCT. A subgroup of patients, namely those with an osteoclast-poor form of ARO, caused by mutations in the TNFSF11 (tumor necrosis factor ligand superfamily, member 11, also known as receptor activator of NF-kB ligand, RANKL) gene, cannot be treated with HSCT.

This study tested a hypothesis that administration of exogenous soluble recombinant murine RANKL (sRANKL) could be used in the treatment of RANKL-associated ARO. RANKL is a cytokine driving osteoclast progenitors to differentiate along the osteoclast lineage. This study used osteopetrotic $R A N K L$ knockout mice (Rankl ${ }^{--}$), which closely resemble the human condition. The data indicate that systemic administration of sRANKL to Rankl ${ }^{---}$mice for 1 month rescued their bone defects and had beneficial effects on hematopoietic organs. On the other hand, overtreatment caused major adverse effects, highlighting the importance of careful dose titration and monitoring if applied to humans. RANKL has been a focus of active research for several years - and usually regarded as a harmful over- 
actively functioning target for therapy. This research has led to development of denosumab, a fully human monoclonal antibody that binds to RANKL and prevents its function. Denosumab is today widely used in the treatment of postmenopausal osteoporosis and cancer-induced skeletal lesions. This study shows that a fine balance is needed within the RANKL-associated skeletal signaling network - severe problems arise when too much or too little RANKL is present.

\title{
New therapies \\ Further indications for denosumab
}

\author{
Shroff R, Beringer O, Rao K, Hofbauer LC, Schulz A \\ Great Ormond Street Hospital for Children, London, UK \\ rukshana.shroff@gosh.nhs.uk \\ N Engl J Med 2012;367:1766-1767
}

Denosumab for posttransplantation hypercalcemia in osteopetrosis

Background: Hematopoietic stem-cell transplantation (HSCT) is the only cure for osteopetrosis, a heterogeneous group of heritable disorders with defective osteoclast-mediated bone resorption. Restitution of osteoclast function after HSCT can be associated with severe hypercalcemia.

Methods: The authors describe effects of denosumab treatment during posttransplantation hypercalcemia in 2 children with osteopetrosis due to a homozygous loss-of-function mutation in the TNFRSF11A gene.

Results: Prior to HSCT, both patients, aged 12 and 3 years, had low serum calcium and elevated parathyroid hormone levels despite oral supplementation. Severe hypercalcemia developed in both patients within 12 days after HSCT; they also developed nephrocalcinosis. Therapeutic attempts with alkaline diuresis, calcitonin, methylprednisolone, intravenous pamidronate, and dialysis were unsuccessful to control hypercalcemia. Patient 1 received denosumab, a monoclonal antibody to RANK ligand, subcutaneously on day 324 after HSCT, when her serum calcium was $3.6 \mathrm{mmol} / \mathrm{l}$ despite hemodialysis. Normocalcemia and resolution of symptoms were observed within 2 days. Continued therapy effectively controlled hypercalcemia and her kidney function normalized. In patient 2 a similar rapid normalization of calcium levels was observed within $12 \mathrm{~h}$ of denosumab treatment. The patient however developed severe pulmonary hypertension and died 6 weeks later.

Conclusion: Denosumab is an established therapy for osteoporosis and bone metastases, and it rarely causes hypocalcemia. Denosumab therapy may also control hypercalcemia after HSCT in patients with osteopetrosis.

This short report by Shroff and colleagues shows a surprisingly rapid improvement of hypercalcemia with denosumab, a fully humanized monoclonal RANK ligand (RANKL) antibody, in 2 children who had undergone hematopoietic stem-cell transplantation for severe osteopetrosis. Posttransplantation hypercalcemia was related to recovery of osteoclast function in a situation where bone mass is greatly increased. A significant advantage in these patients, and possibly even more widely in pediatric patients, is the short half-life of denosumab. The humanized antibody degrades within 3-4 months after injection and is not stored in the body [10], whereas bisphosphonates remain in the skeleton for years and may also impair osteoclast function for longer than desired. In last year's chapter [11], we reported on the first pediatric study using denosumab in a patient with severe fibrous dysplasia. Another study recently described short-term treatment results with denosumab in 4 patients, aged between 5.7 and 18.5 years, with osteogenesis imperfecta (OI) type VI [12]. This form of $\mathrm{OI}$ is autosomal recessive and caused by mutations in SERPINF1. Experimental evidence suggests that loss of functional SERPINF1 leads to osteoclast activation via the RANK/RANKL pathway. The 4 children with OI type VI had shown continuously elevated bone resorption markers during preceding bisphosphonate treatment. Denosumab was injected subcutaneously at a dose of $1 \mathrm{mg} /$ $\mathrm{kg}$ body weight every 3 months, similar to studies on adult osteoporosis. Denosumab was well tolerated, over the follow-up time of 5-33 weeks, the patients and their parents reported no side effects. One patient suffered a radiologically confirmed fracture after a mild trauma but fracture healing 
was normal. Laboratory parameters suggested that the treatment reversibly reduced bone resorption and the authors speculated that denosumab may be a therapeutic option for patients with OI type VI and possibly even in other types of OI. Further studies, in controlled study settings, are urgently needed to assess the safety and long-term treatment effects of denosumab in various pediatric indications, including hypercalcemia, primary and secondary osteoporosis and various rare skeletal disorders.

\section{New swings in the wingless pathway}

\section{WNT1 mutations in early-onset osteoporosis and osteogenesis imperfecta}

Laine CM, Joeng KS, Campeau PM, Kiviranta R, Tarkkonen K, Grover M, Lu JT, Pekkinen M, Wessman M, Heino TJ, Nieminen-Pihala V, Aronen M, Laine T, Kroger H, Cole WG, Lehesjoki AE, Nevarez L, Krakow D, Curry CJ, Cohn DH, Gibbs RA, Lee BH, Mäkitie O

Folkhälsan Institute of Genetics, University of Helsinki, Helsinki, Finland

N Engl J Med 2013;368:1809-1816

Background: Despite high prevalence and heredity of osteoporosis, only a few underlying genes are known. Phenotypes resulting from LRP5 and LRP6 mutations have confirmed the significance of the winglesstype (WNT) signaling pathway for skeletal homeostasis.

Methods: The authors ascertained a family with dominantly inherited early-onset osteoporosis, characterized by low bone turnover, reduced bone mineral density, and vertebral compression fractures, and another family with recessive osteogenesis imperfecta.

Results: The study identified by linkage analysis and exome sequencing disease-causing mutations in the WNT1 gene in both families. Ten family members with dominantly inherited early-onset osteoporosis had a heterozygous missense mutation, c.652T $\rightarrow G$ (p.Cys218Gly). In the family with 2 siblings affected by recessive osteogenesis imperfecta, the authors identified a homozygous nonsense mutation, c. $884 \mathrm{C} \rightarrow \mathrm{A}$, p.Ser $295^{*}$. In vitro, aberrant forms of the WNT1 protein showed impaired capacity to induce canonical WNT signaling, their target genes, and mineralization. In mice, Wnt1 was clearly expressed in bone marrow, especially in B-cell lineage and hematopoietic progenitors but not in osteoblasts or osteoclasts, suggesting that hematopoietic cell-derived WNT1 regulates bone formation. Expression of the gene was seen also in a subset of osteocytes. These findings suggested the presence of altered cross-talk in WNT signaling between the hematopoietic and osteoblastic lineage cells.

Conclusion: This report identifies human skeletal diseases associated with mutations in WNT1. The severity of osteoporosis underscores the importance of WNT1 in bone metabolism.

\section{Mutations in WNT1 cause different forms of bone fragility}

Keupp K, Beleggia F, Kayserili H, Barnes AM, Steiner M, Semler O, Fischer B, Yigit G, Janda CY, Becker J, Breer S, Altunoglu U, Grunhagen J, Krawitz P, Hecht J, Schinke T, Makareeva E, Lausch E, Cankaya T, Caparros-Martin JA, Lapunzina P, Temtamy S, Aglan M, Zabel B, Eysel P, Koerber F, Leikin S, Garcia KC, Netzer C, Schonau E, Ruiz-Perez VL, Mundlos S, Amling M, Kornak U, Marini J, Wollnik B

Institute of Human Genetics, University Hospital Cologne, University of Cologne, Center for Molecular Medicine Cologne, University of Cologne, and Cologne Excellence Cluster on Cellular Stress Responses in Aging-Associated Diseases, University of Cologne, Cologne, Germany

Am J Hum Genet 2013;92:565-574

Background: Osteogenesis imperfecta (OI) is a monogenic disease with increased bone fragility and low bone mass. The phenotypic spectrum ranges from very mild to severe and lethal types of the disease. The majority of OI cases are caused by mutations in COL1A1 and COL1A2, encoding the two $\alpha$ chains of collagen type I. Autosomal recessive inheritance has been described in nearly $10 \%$ and several causative mutations have been identified in genes encoding mainly proteins involved in collagen type I folding, modification, or matrix mineralization.

Methods: This study used exome sequencing to identify disease-causing gene defects in consanguineous families with recessive OI. 
Results: The authors show that hypofunctional alleles of WNT1 cause autosomal recessive OI. In consanguineous families, they identified five homozygous mutations in WNT1: one frameshift mutation, two missense mutations, one splice-site mutation, and one nonsense mutation. In addition, in a family affected by dominantly inherited early-onset osteoporosis, a heterozygous WNT1 missense mutation was identified in affected individuals. Functional analyses showed that altered WNT1 proteins failed to activate canonical LRP5-mediated WNT-regulated $\beta$-catenin signaling. Osteoblasts cultured in vitro showed enhanced Wnt1 expression with advancing differentiation, indicating a role of WNT1 in osteoblast function and bone development.

Conclusion: The findings confirm that homozygous and heterozygous variants in WNT1 predispose to low-bone-mass phenotypes of variable severity. The authors speculate that the findings can be useful for development of more effective therapeutic strategies for congenital forms of bone fragility, as well as for common forms of age-related osteoporosis.

\section{WNT1 mutations in families affected by moderately severe and progressive recessive osteogenesis imperfecta}

Pyott SM, Tran TT, Leistritz DF, Pepin MG, Mendelsohn NJ, Temme RT, Fernandez BA, Elsayed SM, Elsobky E, Verma I, Nair S, Turner EH, Smith JD, Jarvik GP, Byers PH

Department of Pathology, University of Washington, Seattle, WA, USA

shawnap@uw.edu

Am J Hum Genet 2013;92:590-597

Background: Osteogenesis imperfecta (OI) is a heritable disorder that ranges in severity from death in the perinatal period to an increased lifetime risk of fracture. Mutations in COL1A1 and COL1A2 result in dominant forms of OI, and mutations in several other genes result in recessive forms of OI.

Methods: Exome sequencing was used in four families with recessive OI to detect the causative gene mutations.

Results: The authors describe four recessive OI-affected families with disease-causing mutations in wingless-type MMTV integration site family 1 (WNT1). Affected individuals had in family 1, a homozygous missense mutation; in family 2 , a homozygous nonsense mutation predicted to produce truncated WNT1; in family 3, a nonsense mutation and a single-nucleotide duplication on different alleles, and in family 4, a homozygous 14-bp deletion. The mutations in families 3 and 4 are predicted to result in nonsense-mediated mRNA decay and absence of WNT1.

Conclusion: Biallelic loss-of-function mutations in WNT1 result in a clinical picture that includes bone fragility with a moderately severe and progressive presentation that is not easily distinguished from dominant OI type III.

Osteoporosis, the most common skeletal disorder in the Western world, is characterized by low bone mineral density, impaired bone quality, and fragility fractures. Genetic factors play an important role, but the main contributing genes remain largely unknown. The role of the canonical wingless-type (WNT) pathway, which in bone promotes osteogenesis, has been extensively studied since the identification of the low-density lipoprotein receptor-related protein (LRP) 5 mutations in both high and low bone mass phenotypes a decade ago [13]. LRP5 functions as a co-receptor to Frizzled and mediates signal transduction of the WNT ligands. Biallelic loss-of-function mutations in LRP5 result in the recessive osteoporosis-pseudoglioma syndrome with low bone mass and blindness, whereas heterozygous gain-of-function mutations result in various high bone mass phenotypes. Mouse genetics have confirmed the importance of canonical Wnt signaling in the regulation of bone homeostasis, with activation of the pathway leading to increased, and inhibition leading to decreased, bone mass. The importance of WNT signaling has also been highlighted in numerous genome-wide association studies (although none of them has picked up WNT1). The pathway is now the target for therapeutic interventions to develop new effective osteoporosis therapies [14].

The key WNT ligand in the skeleton has remained unknown - until now. Previous studies have suggested that several of the 19 known WNTs participate in skeletal homeostasis. However, the findings in these three recent studies provide evidence that WNT1 is the major ligand and activator of the WNT-signaling cascade in bone tissue. The studies independently show that biallelic or heterozygous loss-of-function mutations in WNT1 lead to compromised bone mass and strength, the severity being dose-dependent: when both alleles are non-functioning, the phenotype resembles severe OI with 
prenatally or infancy-onset skeletal disease, whereas heterozygous mutations lead to milder, yet clinically very significant, osteoporosis. Interestingly, studies on Wnt1 have until now focused on its role in the CNS and in tumor formation. In line with its role in the CNS development, some patients with biallelic mutations did have neurological findings. These extraskeletal features require further studies. This new piece of information - the identity of the long searched for major WNT ligand - takes us a major leap forward in the skeletal field.

\title{
Food for thought
}

\section{Dancing with sex hormones, could iron contribute to the gender difference in osteoporosis?}

\author{
Huang $X, X u Y$, Partridge NC \\ Division of Rheumatology, Department of Medicine, New York University (NYU) School of Medicine, NYU Hospital \\ for Joint Diseases, New York, NY, USA \\ Xi.Huang@nyumc.org \\ Bone 2013;55:458-460
}

Background: Women are at a greater risk of developing osteoporosis than men, but the reasons for the gender differences are incompletely understood. Skeletal traits are highly heritable and many common single nucleotide polymorphisms have pointed to genes accounting for variability in BMD and fracture risk. However, no gender differences in the effects of autosomal gene polymorphisms on BMD were found in separate analyses of more than 50,000 men and women. Therefore, postnatal factors should be considered when looking for potential causes for gender differences in the prevalence of osteoporosis.

Methods: The authors of this commentary have reviewed current literature and looked for factors that might have a role in gender differences in age-related changes in bone mass and in the greater propensity of females to develop osteoporosis.

Results: Several lines of evidence suggest that body iron stores may play a role in the development of osteoporosis. Pathological iron overload has an inhibitory effect on bone formation and results in decreased bone mineral density; further, high serum ferritin levels correlate with accelerated bone loss. While in premenopausal women iron deficiency is common due to monthly blood loss, the menopausal transition leads to an increase in body iron stores concurrently with the decrease in estrogen levels. Estrogen decreases by $90 \%$ during menopausal transition, and levels of serum ferritin, an iron storage protein and an indicator of body iron status, increase 2- to 3-fold from premenopause to postmenopause. In males, in contrast, iron levels increase in conjunction with the increase in testosterone from 20 to 40 years of age, then reverse between ages 40 and 50 years and gradually decrease in tandem thereafter. The decreased bone formation caused by increased iron, coupled with enhanced bone resorption by estrogen deficiency, could accelerate bone loss in women. In older men, however, decreasing levels of testosterone coincide with decreasing levels of iron and the net effect of these two antagonizing factors is not as detrimental to the bone mass as high iron and low estrogen in women.

Conclusion: The authors conclude that together the data from animal and human studies support a hypothesis that it is the combined effects of decreased sex hormones and increased iron that orchestrate bone loss in both postmenopausal women and middle-aged men, the net effect being greater bone loss and suppression of bone formation in women, with consequent increase in the risk of osteoporosis.

Iron is becoming an interesting new player in the bone field. The detrimental effects of iron on bone metabolism have been shown in studies evaluating conditions with pathological iron overload due to e.g. frequent transfusions in patients with thalassemia. Although the pathogenesis of bone loss even in these patients is multifactorial, the contributing factors involving various endocrine dysfunctions such as delayed sexual maturation, diabetes, hypothyroidism, growth hormone deficiency or parathyroid gland dysfunction, direct iron toxicity on osteoblasts is likely to play a significant role [15]. More recent studies have further dissected the effects of iron on bone metabolism and osteocyte function. A study in mice suggested that iron deficiency stimulates transcription of FGF23, a phosphaturic factor, in osteocytes [16]. In another study, Wolf et al. [17] examined the effects of iron defi- 
ciency and its rapid correction with intravenous iron on FGF23 levels in 69 women with iron deficiency anemia secondary to heavy uterine bleeding. Their randomized study showed for the first time in humans that iron deficiency anemia is associated with markedly elevated cFGF23 levels and that rapid correction of iron deficiency with intravenous iron preparations reduced cFGF23 levels by approximately $80 \%$ within $24 \mathrm{~h}$. Together, these studies suggest that iron status is likely to influence osteoblast and osteocyte function, mineral homeostasis, and, as speculated by Huang and co-authors, the development of osteoporosis. However, the data remain scarce and partly controversial; further studies are clearly needed to clarify the role of iron in skeletal homeostasis.

\title{
New genes \\ A new skeletal dysplasia family with hypoparathyroidism
}

\section{FAM111A mutations result in hypoparathyroidism and impaired skeletal development}

\author{
Unger S, Gorna MW, Le Bechec A, Do Vale-Pereira S, Bedeschi MF, Geiberger S, Grigelioniene G, Horemuzova E, \\ Lalatta F, Lausch E, Magnani C, Nampoothiri S, Nishimura G, Petrella D, Rojas-Ringeling F, Utsunomiya A, Zabel B, \\ Pradervand S, Harshman K, Campos-Xavier B, Bonafe L, Superti-Furga G, Stevenson B, Superti-Furga A \\ Department of Pediatrics, Lausanne University Hospital, University of Lausanne, and Medical Genetics Service, \\ Lausanne University Hospital, University of Lausanne, Lausanne, Switzerland \\ Am J Hum Genet 2013 May 14 (E-pub ahead of print)
}

Background: Kenny-Caffey syndrome (KCS) and the similar but more severe osteocraniostenosis (OCS) are genetic conditions characterized by impaired skeletal development with small and dense bones, short stature, and primary hypoparathyroidism with hypocalcemia. The pathogenetic mechanisms underlying the diseases have remained unknown.

Methods: This study involved 5 individuals with KCS and 5 with OCS. An exome-sequencing approach was used to detect the disease-causing gene defects.

Results: Whole-exome data on 4 affected individuals and subsequent direct sequencing in the others showed heterozygous de novo mutations in the FAM111A gene in all patients with KCS and OCS. One mutation was identified in 4 individuals with KCS, and another mutation was identified in 2 individuals with OCS. The findings confirm that OCS and KCS are autosomal dominant conditions and allelic disorders of different severity. FAM111A encodes a protein with homology to trypsin-like peptidases; the native function of the protein is unknown. Molecular modeling of FAM111A showed that residues affected by KCS and OCS mutations are clustered on a segment of the protein at or close to its outer surface, suggesting that the pathogenesis involves the interaction with unidentified partner proteins. Conclusion: The study confirms FAM111A gene mutations as the cause for KCS and OCS. FAM111A appears to be crucial to a pathway involved in parathyroid hormone production, calcium homeostasis, and skeletal development and growth.

Kenny-Caffey syndrome (KCS) is characterized by short stature, thin long bones with narrow diaphyses, recurrent hypocalcemia with low parathyroid hormone concentration requiring treatment with active vitamin $D$, delayed closure of fontanels, defective dentition, small eyes with hypermetropia, and frontal bossing with a triangular face. Osteocraniostenosis (OCS) is a perinatally lethal condition with remarkably similar but more severe features, including gracile bones with thin diaphyses and constriction of the medullary cavity, microphthalmia, and premature closure of basal cranial sutures. The authors have successfully phenotyped their relatively small patient cohort and used wholeexome sequencing approach to detect the disease-causing mutations. Interestingly, these were found to reside in the FAM111A gene, which has not previously been associated with the skeleton or endocrine functions. The authors provide no functional data and the disease mechanisms remain unknown. In OCS, histological evaluation of the skeleton showed that the overall growth plate architecture was preserved, the chondrocyte columns were slightly shorter and plumper than normal but no major abnormalities were present. The diaphyseal cortical bone was thicker than normal. Bone trabeculae were thicker and lacunae reduced. Osteoid lamellae had an unusually wavy appearance. 
These findings correlate well with skeletal features in PTH-deficient mice [18]. It will be interesting to learn more about the molecular mechanisms leading from the gene mutations to the phenotype and particularly about the role of parathyroid hormone therein. Further, an intriguing thought is that the phenotypic variability of the disorders caused by FAM111A mutations may be even greater and include in addition to KCS and OCS also children with hypoparathyroidism and relatively mild skeletal phenotype.

\title{
Food for thought \\ Physical activity brings sustained improvement to bone health in children
}

\section{Three-year follow-up results of bone mineral content and density after a school-based physical activity randomized intervention trial}

\author{
Meyer U, Ernst D, Zahner L, Schindler C, Puder JJ, Kraenzlin M, Rizzoli R, Kriemler S \\ Swiss Tropical and Public Health Institute, and University of Basel, Basel, Switzerland, and Department of Human \\ Movement Science, Maastricht University, Maastricht, The Netherlands \\ ursina.meyer@maastrichtuniversity.nl \\ Bone 2013;55:16-22
}

Background: Physical activity is known to positively influence bone mass accrual during growth and can therefore be used in osteoporosis prevention. The authors have previously shown that a 9-month general school-based physical activity intervention increased bone mineral content (BMC) and density $(\mathrm{aBMD})$ in primary school children. However, it was not known whether these beneficial short-term effects would persist and therefore $\mathrm{BMC}$ and $\mathrm{aBMD}$ were measured again 3 years after cessation of the intervention.

Methods: All children from the intervention group (INT, $\mathrm{n}=297$ ) and the control group (CON, $\mathrm{n}=205$ ) who had participated in KISS (Kinder- und Jugendsportstudie) were contacted 3 years later. The intervention comprised of daily physical education with daily impact loading activities over 9 months. Anthropometry, vigorous physical activity (VPA) measured by accelerometers and BMC/aBMD for total body, femoral neck, total hip, and lumbar spine by dual-energy X-ray absorptiometry (DXA) were measured. Sex- and age-adjusted Z-scores of BMC or aBMD at follow-up were regressed on intervention ( 1 vs. 0 ), the respective Z-score at baseline, gender, follow-up height and weight, pubertal stage at follow-up, previous and current VPA, adjusting for clustering within schools.

Results: Altogether $75 \%$ (377/502) of children participated in baseline DXA measurements and of those, $214(57 \%)$ participated in the follow-up. At follow-up the INT group had significantly higher BMC Z-scores at total body $(\mathrm{p}=0.015)$, femoral neck $(\mathrm{p}=0.042)$ and total hip $(\mathrm{p}=0.016)$ and higher aBMD $\mathrm{Z}$-scores for total body $(\mathrm{p}=0.030)$ compared with CON, thus representing $6-8 \%$ higher values. The authors found no differences in the remaining bone parameters. For the subpopulation with baseline VPA ( $\mathrm{n}=163$ ), effect sizes became stronger after baseline VPA adjustment. After adjustment for baseline and current VPA $(\mathrm{n}=101)$, intervention effects were no longer significant, while effect sizes remained the same as without adjustment for VPA.

Conclusion: Beneficial effects of a 9-month general physical activity intervention on BMC appeared to persist over 3 years. However, part of the maintained effects may be explained by current physical activity.

Osteoporosis has been called 'a pediatric disease with geriatric consequences', because the bone mass gained in childhood and adolescence is an important determinant of lifelong skeletal health. Therefore, lifestyle factors during childhood can have a great impact on fracture prevention by maximizing peak bone mass during growth. Although $60-80 \%$ of peak bone mass can be explained by genetic factors [19], also lifestyle factors, such as nutrition and physical activity, are relevant behavioral and modifiable determinants of bone mass in children. Physical activity has been shown to have a positive impact on bone mass in both girls and boys and also during different stages of puberty [20] and various intervention studies have indicated that exercise can improve BMC and bone strength by $1-8 \%$ [21]. The previous study by the same group showed that a general 9-month school-based inter- 
vention program led to an increase of $4.7 \%$ in BMC and $7.3 \%$ in BMD in the lumbar spine [22]. Another more recent study from Sweden reported that an intense 4-year exercise program led to a mean of $7 \%$ annual gain in lumbar spine BMC and that the exercise did not increase the fracture risk [23]. However, it is not known whether such effects could persist over extended time periods and ideally up to adulthood.

Therefore, Meyer et al. [22] now performed a follow-up of their previous study and measured bone variables 3 years after the end of their previous intervention program. In the follow-up, they were able to demonstrate that the positive effects on bone were partially maintained over 3 years. It is not surprising, however, that part of the maintained effects were explicable by the current amount of children's physical activity. Whereas the short-term effects of their intervention program showed significant benefits on $B M C$ and $A B M D$, the long-term effects were mainly observed for BMC. Since the size dependency of BMC and BMD is especially relevant in the three-dimensional growth of pediatric bone, future studies addressing bone structure are needed to understand how the intervention truly affects the bone maintenance. Meanwhile, we must encourage our children to jump, run and climb - practicing regular physical activity in prepubertal ages and continuing into puberty is a promising strategy to build bones and reduce fracture risk in later life.

\section{New therapies \\ New tools to combat excessive FGF23}

\section{Pharmacological inhibition of fibroblast growth factor (FGF) receptor signaling ameliorates FGF23-mediated hypophosphatemic rickets}

Wohrle S, Henninger C, Bonny O, Thuery A, Beluch N, Hynes NE, Guagnano V, Sellers WR, Hofmann F, Kneissel M, Graus Porta D

Novartis Institutes for BioMedical Research, Basel, Switzerland

J Bone Miner Res 2013;28:899-911

Background: In kidney, circulating fibroblast growth factor 23 (FGF23) inhibits phosphate reabsorption and leads to decreased synthesis and enhanced catabolism of 1,25 -dihydroxyvitamin $\mathrm{D}_{3}\left(1,25[\mathrm{OH}]_{2} \mathrm{D}_{3}\right)$ hence modulating phosphate metabolism. Several hereditary hypophosphatemic disorders with skeletal abnormalities, including X-linked hypophosphatemic rickets (XLH) and autosomal recessive hypophosphatemic rickets (ARHR), are associated with raised levels of FGF23, renal phosphate wasting and decreased $1,25(\mathrm{OH})_{2} \mathrm{D}_{3}$ levels. Current therapies for these diseases are restricted to vitamin $\mathrm{D}$ analogues and phosphate supplements, often merely resulting in partial correction of the skeletal aberrations. This study aimed to evaluate the use of a novel selective, pan-specific FGFR inhibitor for the treatment of hypophosphatemic disorders associated with high FGF23 levels.

Methods: Administration of oral NVP-BGJ398 in two different hypophosphatemic mouse models, Hyp and Dmp1-null mice, resembling the human diseases XLH and ARHR.

Results: Pharmacological inhibition of FGFRs counteracted FGF23 signaling and rescued the hypophosphatemic and hypocalcemic conditions of Hyp and Dmp1-null mice. In addition, FGFR inhibition improved skeletal growth, bone mineralization and rickets in Hyp mice.

Conclusion: The authors propose NVP-BGJ398 treatment as an alternative for the therapy of FGF23mediated hypophosphatemic rickets.

Tremendous progress has been made in several areas since the discovery of FGF23 less than 15 years ago: (1) description of its mode of action and requirement of the co-receptor KLOTHO to activate FGF receptors (FGFRs), (2) genetic characterization of most forms of hereditary hypophosphatemic rickets, (3) generation of mouse models recapitulating human diseases associated with FGF23 signaling excess or deficiency, and (4) production of new therapies targeting FGF23 action or signaling for the cure of hypophosphatemic conditions.

In the present study, Wohrle and colleagues have taken advantage of the specific pan-FGFR inhibiting actions of the NVP-BGJ398 compound, initially developed as an anti-cancer drug, to counteract FGFR signaling in mice models of hypophosphatemia and increased levels of Fgf23. Their study was moti- 
vated by the insufficient correction of rickets and growth defect with the currently available therapy with vitamin $D$ analogues and phosphate supplements. Very interestingly, in Hyp mice, which is the historical model of X-linked hypophosphatemic rickets (XLH), inhibition of FGF signaling through NVP-BGJ398 restored most of the consequences of FGF23 excess, including hypophosphatemia, suppressed $1,25(\mathrm{OH})_{2}$ vitamin $\mathrm{D}$ production, defective weight gain, increased PTH, osteomalacia with increased osteoid surface within the bone, and rickets. Despite a partial effect on longitudinal bone growth, and likewise anti-FGF23 antibodies, this study opens a novel therapeutic area. The results give hope to patients with phosphate wasting due to excessive FGF23 signaling including conditions such as $\mathrm{XLH}$, autosomal dominant hypophosphatemic rickets due to mutations in FGF23, tumor-induced osteomalacia or autosomal recessive hypophosphatemic rickets due to DMP1 mutations. However, several issues need to be solved before translating into human applications in these rare diseases, especially in children. First, studies are needed to evaluate the impact of FGFR inhibition on other important biological processes such as angiogenesis or development. Second, FGFR inhibition promotes further increase in circulating FGF23 levels and may hence foster off-target effects of FGF23. Third, data on long-term evolution of treated animals are awaited to evaluate the therapy's effects on hyperparathyroidism, enthesiopathies or ectopic ossifications that affect patients while they age.

\section{New mechanisms \\ Klotho's complex roles in phosphate homeostasis}

\section{Circulating $\alpha$ Klotho influences phosphate handling by controlling FGF23 production}

Smith RC, O'Bryan LM, Farrow EG, Summers LJ, Clinkenbeard EL, Roberts JL, Cass TA, Saha J, Broderick C, Ma YL, Zeng QQ, Kharitonenkov A, Wilson JM, Guo Q, Sun H, Allen MR, Burr DB, Breyer MD, White KE Biotechnology Discovery Research, Lilly Research Laboratories, Eli Lilly \& Co., Indianapolis, IN, USA J Clin Invest 2012;122:4710-4715

Background: $\alpha$ Klotho is a membrane-bound co-receptor $(\mathrm{mKL})$ of the circulating phosphaturic factor FGF23 and necessary for the intracellular signaling of FGF receptors. Circulating Klotho (cKL) is an endoproteolytic cleavage product of $\mathrm{mKL}$. The authors have previously reported a patient with increased plasma cKL as the result of a translocation $(\mathrm{t}(9 ; 13))$ involving the $\alpha$ KLOTHO gene; the patient presented with rickets and a complex endocrine profile, including paradoxically elevated plasma FGF23, despite hypophosphatemia. This study evaluated the hypothesis that cKL regulates phosphate handling through control of FGF23 expression.

Methods: Elevated cKL levels were obtained in mice through injection of an adeno-associated virus producing cKL.

Results: Treated animals showed: (i) dose-dependent hypophosphatemia and hypocalcemia, with markedly elevated FGF23 (38- to 456-fold), (ii) fractures and reduced bone mineral content, (iii) expanded growth plates and severe osteomalacia, and (iv) highly increased bone Fgf23 mRNA (>150-fold). cKL activity in vitro was specific for interactions with FGF23 and was FGF receptor-dependent.

Conclusion: cKL potently stimulates FGF23 production in vivo, which is consistent with the phenotype of the patient with the translocation involving the $\alpha$ KLOTHO gene.

Klotho was first characterized as an anti-aging agent, as klotho-deficient mice exhibited a reduced life-span. In 2006, Urakawa et al. [24] identified Klotho through proteomic approach as a co-receptor of FGF receptors, and showed it to be necessary for the intracellular activation of ERK signaling pathway. It provided pertinent insights into the diversity of biological responses generated by receptor systems like FGFRs. Brownstein et al. [25] reported in a 2008 issue of PNAS on a carefully investigated young girl who at 13 months presented with severe rickets, fractures, hypophosphatemia due to phosphate wasting, dramatically elevated PTH $(1,233 \mathrm{pg} / \mathrm{ml}, \mathrm{nl} 10-60)$, low 1,25(OH) 2 vitamin D and elevated FGF23 (>12-fold the upper limit of normal). The phenotype differed from that of X-linked hypophosphatemic rickets by the severity of bone demineralization, the early onset of hyperparathyroidism, and the extremely elevated FGF23 levels. Convincingly, the authors attributed the pheno- 
type to a de novo translocation between chromosomes 9 and 13, which lead to increased serum $\alpha$ Klotho [25]. Following this report it became obvious that Klotho is critical for phosphate handling and the modulation of PTH secretion.

In this study, the authors demonstrated that, unexpectedly, the circulating and soluble form of Klotho (cKL, cleaved from $\alpha$ Klotho) robustly increased Fgf23 mRNA and protein expression through Fgf23-FGF signaling in the bone. They had thereby drawn a loop where cKL promotes Ffg23 production through FGFR signaling, which in turn activates Fgf23 signaling (in bone and kidney) through FGFRs. Fgf23 expression is sustained and leads to hypophosphatemia. This is consistent with the phenotype observed in patients with osteoglophonic dysplasia, a rare chondrodysplasia caused by activating mutations in the FGFR1-3 receptors. The patients present with hypophosphatemia due to phosphate wasting and, until today, inexplicably elevated Fgf23 levels (hypophosphatemia not driven by FGF23 suppresses Fgf23 expression) [26]. CKL appears as a strong regulator of Fgf23 expression. There is no doubt that it will be necessary to further evaluate its contribution to the physiology and pathophysiology of phosphate handling.

\title{
New mechanism \\ FHH caused by defective CaSR endocytosis
}

\author{
Mutations in AP2S1 cause familial hypocalciuric hypercalcemia type 3 \\ Nesbit MA, Hannan FM, Howles SA, Reed AA, Cranston T, Thakker CE, Gregory L, Rimmer AJ, Rust N, Graham U, \\ Morrison PJ, Hunter SJ, Whyte MP, McVean G, Buck D, Thakker RV \\ Academic Endocrine Unit, Nuffield Department of Clinical Medicine, University of Oxford, Oxford, UK \\ Nat Genet 2013;45:93-97
}

Background: Adaptor protein-2 (AP2) is critical for clathrin-mediated endocytosis, which internalizes plasma membrane constituents such as G-protein-coupled receptors, including the calcium-sensing receptor (CaSR). AP2 links clathrin to vesicle membranes and binds to dileucine-based motifs of membrane-associated cargo proteins.

Methods: The authors used exome capture and high-throughput sequence analysis of genomic DNA in two unrelated kindreds with familial hypocalciuric hypercalcemia type 3 (FHH3) to define the underlying cause. Direct sequencing of the gene was subsequently performed on 50 unrelated individuals with FHH in whom CASR mutations had been excluded.

Results: The study identified AP2S1 (coding for AP2 sigma subunit) as a putative causative gene for the disease. In vitro, AP2S1 mutations decreased the sensitivity of CaSR-expressing cells to extracellular calcium and reduced CaSR endocytosis, probably through loss of interaction with cargo proteins, whose disruption also decreased intracellular signaling.

Conclusion: AP2 appears as a key regulator for extracellular calcium homeostasis.

FHH or familial hypocalciuric hypercalcemia is characterized by lifelong hypercalcemia, inappropriately normal or elevated PTH and low urinary excretion of calcium. Heterozygous loss of function mutations in the calcium-sensing receptor gene (CaSR) were identified in 1993 as the major cause of FHH [27]. Upon ligand $\left(\mathrm{Ca}^{2+}\right)$ binding, through activation of the $\mathrm{G}$ protein $\alpha$-subunit and phospholipase $C$, activation of the CaSR ultimately prevents PTH secretion in the parathyroid gland cells and urinary calcium absorption in the kidney. In some families however, no mutations in the CaSR were found.

In 1999, the authors used genomic linkage analysis in a very large FHH family from Oklahoma and identified a disease-linked region in chromosome 19q13 [28]. The development of exome capture and high throughput sequencing allowed them to investigate the 110 genes of the narrowed candidate region and eventually pinpoint disease-causing recurrent amino acid substitutions in the AP2S1 gene in this large kindred and 10 other unrelated, similarly affected individuals or families. The type 3 of $\mathrm{FHH}$, initially termed 'Oklahoma variant', is thus linked to mutations in AP2S1. The calcium homeostasis phenotype described by Nesbit and colleagues is strikingly similar to that of patients bearing mutations in the CaSR. The authors have carefully deciphered the impact of the mutations 
on CaSR signaling and PTH secretion. The introduction of the mutation in a cell model significantly decreased the sensitivity of the cell to extracellular calcium thereby proving that the mutant is responsible for the patients' phenotype. However, several aspects remain mysterious. First, although CaSR signaling is impaired in the presence of the mutant AP2S1, its expression is increased at the cell surface. Second, follow-up of the large Oklahoma kindred has shown that FHH3 is a 'benign disease' except from chondrocalcinosis or rare episodes of pancreatitis. It is therefore remarkable that mutations affecting a ubiquitous endocellular system result in a tissue-specific phenotype (parathyroid and kidney). Finally, mutations in AP2S1 were identified in only $25 \%$ of those with FHH and no mutations in CaSR. Other genes thus remain to be discovered, likely partners of this specific intracellular signaling pathway downstream CaSR.

\section{Clinical trials \\ Moving towards evidence-based vitamin D supplementation}

\section{Effect of different dosages of oral vitamin D supplementation on vitamin D status in healthy, breastfed infants: a randomized trial}

Gallo S, Comeau K, Vanstone C, Agellon S, Sharma A, Jones G, L'Abbe M, Khamessan A, Rodd C, Weiler H

School of Dietetics and Human Nutrition, McGill University, Montreal, Que., Canada

JAMA 2013;309:1785-1792

Background: In infancy, vitamin D supplementation is required to support healthy bone mineral accretion and prevent rickets. The proposed optimal 25-hydroxyvitamin $\mathrm{D}(25(\mathrm{OH}) \mathrm{D})$ concentrations for bone health are between 40 and $50 \mathrm{nmol} / 1$; some advocate $75-150 \mathrm{nmol} / \mathrm{l}$. This study investigated the efficacy of different vitamin $\mathrm{D}$ doses in achieving the desired $25(\mathrm{OH}) \mathrm{D}$ concentrations in infants.

Methods: The study was a double-blind randomized clinical trial evaluating plasma $25(\mathrm{OH}) \mathrm{D}$ concentration in 132 healthy, term, breastfed infants, who were randomly assigned to receive oral cholecalciferol (vitamin $\left.\mathrm{D}_{3}\right)$ supplements with $400 \mathrm{IU} /$ day $(\mathrm{n}=39), 800 \mathrm{IU} /$ day $(\mathrm{n}=39), 1,200 \mathrm{IU} / \mathrm{day}(\mathrm{n}=38)$, or $1,600 \mathrm{IU} /$ day $(\mathrm{n}=16)$ from age 1 to 12 months.

Results: By 3 months, 55\% of infants in the 400 -IU/day group, $81 \%$ in the $800-\mathrm{IU} /$ day group, $92 \%$ in the $1,200-\mathrm{IU} /$ day group, and $100 \%$ in the $1,600-\mathrm{IU} /$ day group achieved a $25(\mathrm{OH}) \mathrm{D}$ concentration of $\geq 75 \mathrm{nmol} / \mathrm{l}$. This concentration was not sustained in $97.5 \%$ of infants at 12 months in any of the groups. All dosages led to $25(\mathrm{OH}) \mathrm{D}$ concentrations of $\geq 50 \mathrm{nmol} / \mathrm{l}$ in $97 \%$ of infants at 3 months, and this was sustained in $98 \%$ at 12 months. The 1,600-IU/day dosage was discontinued prematurely because of high plasma $25(\mathrm{OH}) \mathrm{D}$ concentrations.

Conclusions: Only 1,600 IU/day of cholecalciferol increased plasma $25(\mathrm{OH}) \mathrm{D}$ concentration to $75 \mathrm{nmol} / \mathrm{l}$ in $97.5 \%$ of infants. However, this dosage increased $25(\mathrm{OH}) \mathrm{D}$ concentrations to levels that have been associated with hypercalcemia.

In the past year, two intervention studies were carried out aiming at defining the dosage of vitamin D supplementation necessary to reach 'optimal' levels of vitamin D (e.g. those recommended by societies), the study reported above, and the study by Holmlund-Suila et al. [29]. Although designs differed, both studies concluded that vitamin $D$ intake of 1,600 IU/day was required to achieve plasma 25(OH)D concentration of $\geq 75 \mathrm{~nm}$. In addition, Gallo and colleagues demonstrated that with the current standard supplementation of $400 \mathrm{IU} /$ day, only $55 \%$ of the infants reached the 'optimal' $25(\mathrm{OH}) \mathrm{D}$ concentration. The dosing and requirement of vitamin $\mathrm{D}$ supplements or vitamin $\mathrm{D}$ enrichment of dairy products depend on the objective: Is it prevention of rickets and hypocalcaemia or optimization of bone health? Obviously, Gallo et al. have in their 12-month intervention study shown that any vitamin $D$ intake $\geq 400 \mathrm{IU} /$ day sustains plasma levels of $25(\mathrm{OH}) \mathrm{D}>50 \mathrm{~nm}$, hence preventing rickets and allowing bone growth and mineralization. Over a period of 3 months, HolmlundSuila et al. showed that despite relatively high 25(OH)D concentrations, even the dose of 1,600 IU/ day of vitamin D did not modify calcium, phosphorus, PTH or urinary calcium excretion in infants. This is the first step towards identification of the 'optimal' vitamin D supplementation to optimize bone health. 


\section{The osteocyte: an endocrine cell and more}

Dallas SL, Prideaux M, Bonewald LF

Department of Oral and Craniofacial Sciences, School of Dentistry, University of Missouri-Kansas City, MO, USA Endocr Rev 2013 (E-pub ahead of print)

Background: Until the last few years, osteocytes were perceived by many as passive, metabolically inactive bone cells. However, exciting recent discoveries have shown that osteocytes, encased within mineralized bone matrix, are actually multifunctional cells, with many key regulatory roles in bone and mineral homeostasis.

Methods: This review article covers the current literature describing the multiple functions of osteocytes. Results: After the discovery that osteocytes produce circulating fibroblast growth factor 23 (FGF23) that targets the kidney and potentially other organs, bone has been recognized as an important endocrine 'gland'. In addition to serving as endocrine cells and regulators of phosphate homeostasis through FGF23, osteocytes control bone remodeling through regulation of both osteoclasts and osteoblasts, are mechanosensory cells that coordinate adaptive responses of the skeleton to mechanical loading, and also serve as a manager of the bone's reservoir of calcium. Osteocytes survive for decades within the bone matrix, making them one of the longest lived cells in the body.

Conclusion: Osteocytes have multiple key functions in the skeleton. Their viability and survival are therefore extremely important to ensure optimal function of the osteocyte network. When developing new therapeutics, not only the osteoclast and the osteoblast, but even the osteocyte should be considered in new strategies to prevent and treat bone disease.

Interested in skeletal endocrinology? Here is an excellent review article describing how the oncethought-dead osteocyte is gladly vivid and with its multiple functions gradually becoming appreciated as a major gatekeeper in skeletal homeostasis.

References

1. Wilsman NJ, Farnum CE, Leiferman EM, Fry M, Barreto C: Differential growth by growth plates as a function of multiple parameters of chondrocytic kinetics. J Orthop Res 1996;14:927-936.

2. Schipani E, Ryan HE, Didrickson S, Kobayashi T, Knight M, Johnson RS: Hypoxia in cartilage: HIF-1 $\alpha$ is essential for chondrocyte growth arrest and survival. Genes Dev 2001;15:2865-2876.

3. Myllyharju J: Prolyl 4-hydroxylases, key enzymes in the synthesis of collagens and regulation of the response to hypoxia, and their roles as treatment targets. Ann Med 2008;40:402-417.

4. Saito A, Hino S, Murakami T, Kanemoto S, Kondo S, Saitoh M, et al: Regulation of endoplasmic reticulum stress response by a BBF2H7-mediated Sec23a pathway is essential for chondrogenesis. Nat Cell Biol 2009;11:1197-1204.

5. Panda S, Antoch MP, Miller BH, Su AI, Schook AB, Straume M, et al: Coordinated transcription of key pathways in the mouse by the circadian clock. Cell 2002;109:307-320.

6. Yoo SH, Yamazaki S, Lowrey PL, Shimomura K, Ko CH, Buhr ED, et al: PERIOD2::LUCIFERASE real-time reporting of circadian dynamics reveals persistent circadian oscillations in mouse peripheral tissues. Proc Natl Acad Sci USA 2004;101:5339-5346.

7. Hinoi E, Ueshima T, Hojo H, Iemata M, Takarada T, Yoneda Y: Upregulation of per mRNA expression by parathyroid hormone through a protein kinase A-CREB-dependent mechanism in chondrocytes. J Biol Chem 2006;281:2363223642.

8. Yasoda A, Komatsu Y, Chusho H, Miyazawa T, Ozasa A, Miura M, et al: Overexpression of CNP in chondrocytes rescues achondroplasia through a MAPK-dependent pathway. Nat Med 2004;10:80-86.

9. Yasoda A, Kitamura H, Fujii T, Kondo E, Murao N, Miura M, et al: Systemic administration of C-type natriuretic peptide as a novel therapeutic strategy for skeletal dysplasias. Endocrinology 2009;150:3138-3144.

10. Sutjandra L, Rodriguez RD, Doshi S, Ma M, Peterson MC, Jang GR, et al: Population pharmacokinetic meta-analysis of denosumab in healthy subjects and postmenopausal women with osteopenia or osteoporosis. Clin Pharmacokinet 2011;50:793-807.

11. Mäkitie O, Nilsson O: Bone, growth plate and mineral metabolism; in Carel J-C, Hochberg Z (eds): Yearbook of Pediatric Endocrinology 2012. Basel, Karger, 2012.

12. Semler O, Netzer C, Hoyer-Kuhn H, Becker J, Eysel P, Schoenau E: First use of the RANKL antibody denosumab in osteogenesis imperfecta type VI. J Musculoskelet Neuronal Interact 2012;12:183-188.

13. Gong Y, Slee RB, Fukai N, Rawadi G, Roman-Roman S, Reginato AM, et al: LDL receptor-related protein 5 (LRP5) affects bone accrual and eye development. Cell 2001;107:513-523.

14. Baron R, Kneissel M: WNT signaling in bone homeostasis and disease: from human mutations to treatments. Nat Med 2013;19:179-192.

15. Voskaridou E, Terpos E: New insights into the pathophysiology and management of osteoporosis in patients with $\beta$-thalassaemia. Br J Haematol 2004;127:127-139. 
16. Farrow EG, Yu X, Summers LJ, Davis SI, Fleet JC, Allen MR, et al: Iron deficiency drives an autosomal dominant hypophosphatemic rickets (ADHR) phenotype in fibroblast growth factor-23 (Fgf23) knock-in mice. Proc Natl Acad Sci USA 2011;108:E1146-E1155.

17. Wolf M, Koch TA, Bregman DB: Effects of iron deficiency anemia and its treatment on fibroblast growth factor 23 and phosphate homeostasis in women. J Bone Miner Res 2013 (E-pub ahead of print).

18. Miao D, He B, Karaplis AC, Goltzman D: Parathyroid hormone is essential for normal fetal bone formation. J Clin Invest 2002;109:1173-1182.

19. Bonjour JP, Chevalley T, Rizzoli R, Ferrari S: Gene-environment interactions in the skeletal response to nutrition and exercise during growth. Med Sport Sci 2007;51:64-80.

20. Rizzoli R, Bianchi ML, Garabedian M, McKay HA, Moreno LA: Maximizing bone mineral mass gain during growth for the prevention of fractures in the adolescents and the elderly. Bone 2010;46:294-305.

21. Hind K, Burrows M: Weight-bearing exercise and bone mineral accrual in children and adolescents: a review of controlled trials. Bone 2007;40:14-27.

22. Meyer U, Romann M, Zahner L, Schindler C, Puder JJ, Kraenzlin M, et al: Effect of a general school-based physical activity intervention on bone mineral content and density: a cluster-randomized controlled trial. Bone 2011;48:792-797.

23. Lofgren B, Dencker M, Nilsson JA, Karlsson MK: A 4-year exercise program in children increases bone mass without increasing fracture risk. Pediatrics 2012;129:e1468-e1476.

24. Urakawa I, Yamazaki Y, Shimada T, Iijima K, Hasegawa H, Okawa K, et al: Klotho converts canonical FGF receptor into a specific receptor for FGF23. Nature 2006;444:770-774.

25. Brownstein CA, Adler F, Nelson-Williams C, Iijima J, Li P, Imura A, et al: A translocation causing increased $\alpha$-klotho level results in hypophosphatemic rickets and hyperparathyroidism. Proc Natl Acad Sci USA 2008;105:3455-3460.

26. White KE, Cabral JM, Davis SI, Fishburn T, Evans WE, Ichikawa S, et al: Mutations that cause osteoglophonic dysplasia define novel roles for FGFR1 in bone elongation. Am J Hum Genet 2005;76:361-367.

27. Pollak MR, Brown EM, Chou YH, Hebert SC, Marx SJ, Steinmann B, et al: Mutations in the human $\mathrm{Ca}^{2+}{ }^{2+}$ sensing receptor gene cause familial hypocalciuric hypercalcemia and neonatal severe hyperparathyroidism. Cell 1993;75:1297-1303.

28. Lloyd SE, Pannett AA, Dixon PH, Whyte MP, Thakker RV: Localization of familial benign hypercalcemia, Oklahoma variant (FBHOk), to chromosome 19q13. Am J Hum Genet 1999;64:189-195.

29. Holmlund-Suila E, Viljakainen H, Hytinantti T, Lamberg-Allardt C, Andersson S, Makitie O: High-dose vitamin D intervention in infants - effects on vitamin D status, calcium homeostasis, and bone strength. J Clin Endocrinol Metab 2012;97:4139-4147. 